\title{
ABME Special Issue Editorial: Engineering Cell Behavior
}

\author{
Debra Auguste \\ Chemical Engineering, Northeastern University, 360 Huntington Avenue, Interdisciplinary Science and Engineering Center 327, \\ Boston, MA 02115, USA
}

Wilhelm Roux, in 1885, was the first to culture cells ex vivo to study embryological development. He identified cell differentiation, modeled the bifurcation of blood vessels, and described the mechanics of bones, cartilage and tendons. Since then, scientists and engineers have continued to test theories to define the structure-function relationship between mechanical, chemical, and electrical signals and cell behavior. How do cells sense, respond, organize, proliferate, or trigger apoptosis? These questions circumvent the processes of development, repair, regeneration, and combatting disease.

As our knowledge evolves, so has the level of complexity of experimentation. We engineered novel materials that bind specific receptors, control mechanical properties, align cells, and degrade at different rates to direct how cells differentiate, grow, or express genes. We tuned the delivery of molecules to stimulate cell processes or genetically modify gene expression. We designed three-dimensional, complex environments that mimic aspects of physiology. The field continues to progress: Once stagnant systems are now dynamic. Homogeneous materials are yielding to anisotropy. Unicellular systems are now multicellular. We face challenges as the intricacy of experimentation increases; adoption across laboratories, cost, and time.

Our ability to decode nature is still limited. Fundamental molecular analyses and single cell studies aid in identifying components at the microscale. Hierarchical analyses, such as dynamic, multicellular tissue or whole organ analyses, ascertain structural features at the macroscopic level. The connections between a single cell to the organ level are being constructed, from understanding population dynamics, architectural features, transport, materials, and mechanics.

Address correspondence to Debra Auguste, Chemical Engineering, Northeastern University, 360 Huntington Avenue, Interdisciplinary Science and Engineering Center 327, Boston, MA 02115, USA. Electronic mail: d.auguste@northeastern.edu
Over the course of the last 135 years, since Roux cultured the first cell, we have mapped signaling pathways, engineered new materials, built systems that test fluid and solid mechanics, and assessed diseased and healthy tissues. There is still much to discover. Engineering cellular systems lies at the intersection of multiple disciplines: biology, chemistry, material science, biomedical engineering, mechanical engineering, electrical engineering, and medicine. Developing new methods to evaluate live cells in their native environments may inform how we engineer tissues, vessels, and drug delivery vehicles.

Large data sets may help decipher the multitude of signals processed within a cell. This may allow engineers to explore beyond individual pathways to a systems-wide analysis. Interpretation of these large data sets may rely heavily on the use of computational models, machine learning and artificial intelligence. The use of design of experiments methods can reduce the number of trial and error experiments to answer a hypothesis, but artificial intelligence may help deduce solutions to complex problems and machine learning may predict new avenues to achieve a desired goal.

These scientific trajectories all converge on a common purpose. Engineering cell behavior is fundamentally driven by the impact engineers can make in the lives of the people in our communities and families: ameliorating pain in the sick and dying, providing a solution for loss of function in amputees, or overcoming gene dysregulation in patients with cancer. Breakthroughs, to current unsurmountable challenges, may be around the corner. The compilation of articles herein considers new avenues in engineering cell behavior, with aspirations to one day benefit the lives of many people.

Publisher's Note Springer Nature remains neutral with regard to jurisdictional claims in published maps and institutional affiliations. 